\title{
Minimum Distance Pattern Classifiers Based On A New Distance Metric
}

\author{
N. Gaitanis, G. Kapogianopoulos and D.A. Karras \\ Institute of Informatics and Telecommunications,National Research Center "Demokritos", \\ 15310 Aghia Paraskevi, Greece, Fax.:+30 16532175.
}

\begin{abstract}
A method for the design of minimum distance bipolar pattern classifiers based on a new distance metric between the bipolar patterns is described. The new distance metric is defined and its properties are demonstrated. Neural Networks with the Nearest-neighbor recall mechanism [2], trained according to this method, distinguish the images of a prototype pattern $\mathbf{X}^{k}$ from the images of the opponent prototype functions $\mathbf{X}^{q}$, taking into accoun$t$ not only the erroneous pattern elements but also their distinguishing ability. A Neural pattern classifier can be defined by $R$ linear discriminant functions $[1]\left\{F_{1}(\mathbf{X}), . ., F_{R}(\mathbf{X})\right\}$ with $F_{k}(\mathbf{X})=\mathbf{X} * \mathbf{W}^{k}=x_{1} * W_{1}^{k}+x_{2} * W_{2}^{k}+\ldots+x_{n} * W_{n}^{k}$ where, $x_{i}$ are bipolar variables $x_{i}=+1,-1$ and the weights $W_{i}^{k}$ are real numbers and $-1 \leq W_{i}^{k} \leq+1$. In the proposed neural net architecture, the discriminant function $F_{k}(\mathbf{X})$ is then, followed by an output function $T_{k}(F)$ defined as $T_{k}(F)=\left\{\begin{array}{ll}1 & \text { if } F_{k}(\mathbf{X})>1-m N D\left(\mathbf{X}^{k}\right) / 2 \\ -1 & \text { otherwise }\end{array}, k=1, \ldots, R\right.$ and $m N D()$ is the new distance metric defined next. Let $\mathrm{X}^{k}=\left(x_{1}^{k}, x_{2}^{k}, \ldots, x_{n}^{k}\right)$ be a prototype pattern with $k=1,2, \ldots, R$ and $\mathbf{X}=\left(x_{1}, \ldots, x_{n}\right)$ be an unknown pattern. Their Hamming distance $D\left(\mathbf{X}^{k}, \mathbf{X}\right)$ can then be obtained from the Hamming Discriminant Function $H F_{k}(X)=x_{1} * x_{1}^{k}+x_{2} * x_{2}^{k}+\ldots+x_{n} * x_{n}^{k}$ with $W_{i}^{k}=x_{i}^{k}, i=1, \ldots, n$, as $D\left(\mathbf{X}^{k}, \mathbf{X}\right)=\left(H F_{k}\left(\mathbf{X}^{k}\right)-H F_{k}(\mathbf{X})\right) / 2$. Then, in the case of a finite set of $R$ prototype patterns $\mathbf{X}^{k}, k=1, ., R$, a new Discriminant Function $F_{k}(\mathbf{X})=$ $x_{1} * W_{1}^{k}+\ldots+x_{n} * W_{n}^{k}$ can be defined for every pattern $\mathrm{X}^{k}, k=1, ., R$ with respect to its opponent patterns $\mathrm{X}^{q}, q=1, ., R$ and $q \neq k$ as follows:

The weights $W_{i}^{k}, i=1, \ldots, n$ of the Discriminant Function $F_{k}(\mathbf{X})$ are calculated according to the formula

$$
W_{i}^{k}=\left(x_{i}^{k}\right) *\left\{\sum_{q \neq k}\left(1-x_{i}^{k} * x_{i}^{q}\right) /\left(2 * R * D\left(\mathbf{X}^{k}, \mathbf{X}^{q}\right)\right)\right\} .
$$

From the above formula we can see that the minimum value of a weight $W_{i}^{k}$ will be equal to $W_{i}^{k}=0$, in the case where $x_{i}^{k}=x_{i}^{q}$ for every $q=1, \ldots, R$ with $q \neq k$ and the maximum value of the weight $W_{i}^{k}$ when $x_{i}^{k}=-x_{i}^{q}$ for every $q$, will be equal to $W_{i}^{k}=\left(2 * x_{i}^{k}\right) *\left\{\sum_{q \neq k} 1 /(2 *\right.$ $\left.\left.R * D\left(\mathbf{X}^{k}, \mathbf{X}^{q}\right)\right)\right\}$. The properties of the new Discriminant Function can be demonstrated using the above formula of the weights $W_{i}^{k}$ written as $W_{i}^{k}=\left(x_{i}^{k}\right) *\left\{\sum_{q \neq k} 1 /\left(2 * R * D\left(\mathbf{X}^{k}, \mathbf{X}^{q}\right)\right)\right\}-$ $\left\{\sum_{q \neq k}\left(x_{i}^{q}\right) /\left(2 * R * D\left(\mathbf{X}^{k}, \mathbf{X}^{q}\right)\right)\right\}$.

According to the above, the output of the Discriminant Function $F_{k}\left(\mathbf{X}^{k}\right)$ for the pattern $\mathbf{X}^{k}$ will be equal to the maximum value $F_{k}\left(\mathbf{X}^{k}\right)=1$. Also, the output of the Discriminant Function $F_{k}\left(\mathbf{X}^{k^{\prime}}\right)$ for the complement $\mathbf{X}^{k^{\prime}}$ of the prototype pattern $\mathbf{X}^{k}$ will be equal to the minimum value $F_{k}\left(\mathbf{X}^{k^{\prime}}\right)=-1$. Finally, the minimum New Distance $m N D\left(\mathbf{X}^{k}\right)$ between pattern $\mathbf{X}^{k}$ and its opponent patterns $\mathbf{X}^{q}, q=1, \ldots, R, q \neq k$ will be equal to $m N D\left(\mathbf{X}^{k}\right)=1-\max \left\{F_{k}\left(\mathbf{X}^{q}\right)\right\}, q \neq$ $k$.
\end{abstract}

\section{References}

[1] N. Nilsson. Learning Machines. McGraw-Hill, 1965.

[2] P. K. Simpson. Artificial Neural Systems. Pergamon Press, 1990. 